\title{
Analysis of Kytococcus sedentarius Strain Isolated from a Dehumidifier Operating in a University Lecture Theatre: Systems for Aerobic Respiration, Resisting Osmotic Stress, and Sensing Nitric Oxide
}

\author{
Meshari Ahmed Alhadlaq ${ }^{a, b}$ Jeffrey Green ${ }^{a}$ Bassam K. Kudhair ${ }^{c}$

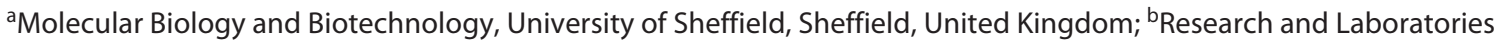 \\ Sector, Saudi Food and Drug Authority, Riyadh, Saudi Arabia; 'Department of Laboratory Investigations, Faculty of \\ Science, University of Kufa, Najaf, Iraq
}

\section{Keywords}

Actinobacteria $\cdot$ Halotolerance $\cdot$ Iron-sulfur cluster $\cdot$ Nitric oxide $\cdot \mathrm{Wbl}$-sigma factor interactions

\begin{abstract}
A strain of Kytococcus sedentarius was isolated from a dehumidifier operating in a university lecture theatre. Genome analysis and phenotypic characterisation showed that this strain, $K$. sedentarius MBB13, was a moderately halotolerant aerobe with a branched aerobic electron transport chain and genes that could contribute to erythromycin resistance. The major compatible solute was glycine betaine, with ectoine and proline being deployed at higher osmolarities. Actinobacteria possess multiple WhiB-like (Wbl) regulatory proteins, and $K$. sedentarius MBB13 has four (WhiB1, WhiB2, WhiB3, and WhiB7). Wbls are iron-sulfur proteins that regulate gene expression through interactions with RNA polymerase sigma factors and/or other regulatory proteins. Bacterial two-hybrid analyses suggested that WhiB1 and WhiB2, but not WhiB3 and WhiB7, interact with the C-terminal domain of the major sigma factor, $\sigma^{\mathrm{A}}$; no interaction was detected between any of the $\mathrm{Wbl}$ proteins and the only alternative sigma factors, $\sigma^{B}, \sigma^{H}$, or $\sigma^{J}$. The interaction between $\sigma^{A}$ and WhiB1 or WhiB2 was disrupted in a heterologous system under growth conditions that produce nitric oxide and the
\end{abstract}

iron-sulfur clusters of the isolated WhiB1 and WhiB2 proteins reacted with nitric oxide. Thus, $K$. sedentarius strain exhibits the major phenotypic characteristics of the type strain and a comprehensive examination of the interactions between its four Wbl proteins and four sigma factors suggested that the Wbl proteins all operate through interaction with $\sigma^{A}$.

(c) 2021 S. Karger AG, Base

\section{Introduction}

Several reports have suggested that human health is likely to be adversely affected by airborne bacteria circulating within the enclosed environment and that the source of these bacteria is mainly human occupants of these spaces (reviewed by Fujiyoshi et al. [2017]). There are two core methods of evaluating the levels of airborne bacteria in an indoor environment. Passive methods expose petri dishes containing solid culture medium to the air. Bacteria that settle from the atmosphere onto the surface of the plate are thus collected and subsequent incubation of the covered plates allows the airborne bacteria collected during the exposure period to be quantified. Alternatively, active methods physically expose liquid or a solid culture media to a known volume of air. Subsequent culturing allows measurement of the bacteria trapped in karger@karger.com

(c) 2021 S. Karger AG, Basel

www.karger.com/mip

Karger ${ }^{\prime}=$
Meshari Ahmed Alhadlaq

Research and Laboratories Sector, Saudi Food and Drug Authority 4904 Northern Ring Branch Road, Hittin District

Riyadh 13513-7148 (Saudi Arabia)

MAHadlaq@sfda.gov.sa 
this way [Napoli et al., 2012]. We were interested in the prevalence of antibiotic-resistant bacteria in the air of a university lecture theatre. To actively sample the air in the lecture theatre, a dehumidifier was prepared so that after operation the airborne bacteria that had collected in the water trap could be cultured. Seven bacterial isolates were identified by $16 \mathrm{~S}$ rRNA gene sequencing and their antibiotic resistance profiles were determined. One of the seven, Kytococcus sedentarius MBB13, was resistant to three antibiotics (ciprofloxacin, erythromycin, and gentamicin) and hence this isolate was subjected to further investigation.

K. sedentarius was first isolated in 1944 from a slide immersed in sea water near San Diego, USA and has since been isolated from human skin and commercial airline cabin air [Osman et al., 2008; Sims et al., 2009]. K. sedentarius is a strictly aerobic, coccoid, gram-positive actinobacterium [Sims et al., 2009]. One characteristic of actinobacteria is the possession of Wbl (WhiB-like) iron-sulfur proteins [Bush, 2018]. The main structural features of $\mathrm{Wbl}$ proteins are the four invariant cysteine residues that coordinate a $[4 \mathrm{Fe}-4 \mathrm{~S}]$ cluster and a conserved five-residue G[V/I]WGG motif [Kudhair et al., 2017; Bush, 2018]. Most actinobacteria possess more than one $\mathrm{Wbl}$ protein. The seven Wbl proteins of Mycobacterium tuberculosis have been extensively studied. WhiB1 is an essential nitric oxide-responsive transcription regulator [Smith et al., 2010; Kudhair et al., 2017]. WhiB2 is also essential and likely has a role in coordinating cell division [Gomez and Bishai, 2000]. WhiB3 has an oxygen- and nitric oxidesensitive [4Fe-4S] cluster and it is thought to be involved in redox homeostasis when $M$. tuberculosis is in a host phagosome and thus is implicated in virulence and persistence [Singh et al., 2007]. The iron-sulfur cluster of WhiB4 is sensitive to oxygen and nitric oxide, and WhiB4 likely regulates the response to oxidative stress because deletion of whiB 4 resulted in over-induction of anti-oxidant activities and enhanced resistance to oxidative stress [Chawla et al., 2012]. The M. tuberculosis whiB5 mutant was attenuated in a mouse model of infection, was unable to resume growth following a chronic infection, and was more sensitive to nitric oxide than the parental strain [Casonato et al., 2012]. WhiB6, is implicated in pathogenesis through its regulation of the virulence critical Type VII secretion system ESX-1 and aspects of aerobic and anaerobic metabolism [Chen et al., 2016]. Lastly, WhiB7 is associated with resistance to several antibiotics that target translation [Lee et al., 2020]. Thus, Wbl proteins are implicated in a wide range of processes that are critical to pathogenesis and evidence suggests that most Wbl pro- teins act as transcriptional regulators that exert their influence on gene expression at least in part through interaction with the housekeeping sigma factor [Burian et al., 2013; Feng et al., 2016; Kudhair et al., 2017; Wan et al., 2020].

Whilst generally considered to be part of the normal human commensal microflora, some strains of K. sedentarius are opportunistic pathogens being associated with pitted keratolysis [Longshaw et al., 2002], haemorrhagic pneumonia in acute myeloid leukaemia patients [Levenga et al., 2004], cerebral cyst, and ventriculoatrial shunt infections for those with congenital hydrocephalus [Greene et al., 1980]. It has been reported that all tested K. sedentarius strains were sensitive to streptomycin, novobiocin, tetracycline, neomycin, vancomycin, and polymyxin B and most were sensitive to erythromycin, kanamycin, and chloramphenicol [Stackebrandt et al., 1995]. The observation that $K$. sedentarius MBB13 was resistant to erythromycin and that topical application of erythromycin is widely used to treat clinical K. sedentarius pitted keratolysis [Pranteda et al., 2014] prompted further investigation of the properties of the K. sedentarius MBB13 isolate.

\section{Materials and Methods}

Bacterial Isolation and Genomic DNA Analysis

A dehumidifier (Meaco 12L Platinum) was operated for $1 \mathrm{~h}$ in a University of Sheffield lecture theatre at the end of the teaching week. Water collected in the dehumidifier was transferred to a 50 $\mathrm{mL}$ Falcon tube and centrifuged. The supernatant was discarded and the liquid that remained $(\sim 50 \mu \mathrm{L})$ was streaked on a Nutrient Agar (NA) plate and incubated at $37^{\circ} \mathrm{C}$ for $18 \mathrm{~h}$. The following day, colonies were sub-cultured on fresh NA plates and were incubated at $37^{\circ} \mathrm{C}$ for $18 \mathrm{~h}$. One colony of each sub-cultured bacterium was inoculated into $5 \mathrm{~mL}$ Nutrient Broth (NB) in $50 \mathrm{~mL}$ Falcon tubes and incubated at $37^{\circ} \mathrm{C}$ for $18 \mathrm{~h}$.

For 16S RNA gene sequencing universal bacterial primers (F27 and R-1492; online supplementary Table 1; for all online suppl. material, see www.karger.com/doi/10.1159/000512751) were used to produce amplified DNA fragments $(\sim 1.5 \mathrm{~kb})$. The DNA sequences of the PCR products were analysed using the BLAST programme available through the National Centre for Biotechnology Information (NCBI) to identify the closest matches in the database. For K. sedentarius MBB13 the match to the reference strain DSM 20547 (541T) was 97\% identical.

Genomic DNA extraction was carried out using either Sigma GenElute or Qiagen QIAamp DNA mini kits according to the manufacturer's instructions. An additional step, to improve cell lysis of $K$. sedentarius MBB13 was treatment of the cell suspensions with lysozyme $(300 \mu \mathrm{g} / \mathrm{mL})$, achromopeptidase $(200 \mu \mathrm{g} / \mathrm{mL})$, lysostaphin $(200 \mu \mathrm{g} / \mathrm{mL})$, mutanolysin $(100 \mu \mathrm{g} / \mathrm{mL})$, and proteinase $\mathrm{K}$ ( $40 \mu \mathrm{L}$ of a $20 \mathrm{mg} / \mathrm{mL}$ solution) for $30 \mathrm{~min}$ at $37^{\circ} \mathrm{C}$ [Sims et al., 2009]. 
Genomic DNA was sequenced by MicrobesNG (University of Birmingham, UK). Four trimmed files (fastq.gz) were used to generate three files for analysis in the ACT software [Carver et al., 2005], namely: (1) Galaxy-[Concatenated_reference_Genbank_ file), (2) Galaxy-[ACT_comparison_file).tabular, and (3) Galaxy[Concatenated_contigs_Genbank_file).

\section{Bacterial Growth}

Bacterial strains and plasmids and their sources are listed in (online supplementary Table 2). K. sedentarius MBB13 cultures were routinely grown aerobically in Luria-Bertani (LB) broth, with orbital shaking $(250 \mathrm{rpm})$ at $37^{\circ} \mathrm{C}$ overnight. The ability to grow in the absence of oxygen was assessed by incubating LB streaked with K. sedentarius $\mathrm{MBB} 13$ in anaerobic jars at $37^{\circ} \mathrm{C}$ for $18 \mathrm{~h}$. In addition, thioglycollate broth was used [Tille, 2014]. Single colonies of K. sedentarius MBB13 or Escherichia coli MG1655 were picked from LB plates and placed into sterilised thioglycollate broth (10 $\mathrm{mL}$ ) in $25 \mathrm{~mL}$ glass universal tubes. The tubes were sealed and incubated at $37^{\circ} \mathrm{C}$ for $48 \mathrm{~h}$.

For preparation of competent cells, overnight cultures $(5 \mathrm{~mL})$ of either E. coli or Mycobacterium smegmatis were grown in LB or 7H9 broth (Sigma-Aldrich), respectively. A $250 \mathrm{~mL}$ flask containing either LB or $7 \mathrm{H} 9(50 \mathrm{~mL})$ was inoculated with $300 \mu \mathrm{L}$ of the overnight culture of E. coli or M. smegmatis. Cultures were incubated at $37^{\circ} \mathrm{C}$ with shaking at $250 \mathrm{rpm}$ until the optical density at $600 \mathrm{~nm}\left(\mathrm{OD}_{600}\right)$ reached $\sim 0.6$ (E. coli, $\left.2 \mathrm{~h}\right)$ or $\sim 0.9$ (M. smegmatis, $\sim 18 \mathrm{~h}$ ). The cultures were immediately placed on ice for $30 \mathrm{~min}$ for E. coli or $\sim 2 \mathrm{~h}$ for M. smegmatis. The bacteria were then collected by centrifugation at $3,500 \mathrm{~g}$ for $10 \mathrm{~min}$ at $4^{\circ} \mathrm{C}$. The supernatant was discarded and the cell pellets were suspended in $10 \%(\mathrm{v} / \mathrm{v})$ cold glycerol and centrifuged at $3,500 \mathrm{~g}$ for $10 \mathrm{~min}$ at $4^{\circ} \mathrm{C}$. This process was repeated twice. The cell pellets were suspended in 1,000 $\mu \mathrm{L} \mathrm{10 \%}$ $(\mathrm{v} / \mathrm{v})$ cold glycerol then, aliquots $(100 \mu \mathrm{L})$ were transferred to precooled Eppendorf tubes in dry ice and stored at $-80^{\circ} \mathrm{C}$.

For initial antibiotic resistance profiling autoclaved ISO agar medium was prepared and left to cool to $\sim 45^{\circ} \mathrm{C}$ before bacterial suspensions were added to match the turbidity of the $0.5 \mathrm{McFar}-$ land standard $\left(0.111 \mathrm{OD}_{600}\right)$. The agar was poured into petri dishes (30 mL per plate) and, after setting, antibiotic discs were placed at the centre of the plates. The plates were incubated at $37^{\circ} \mathrm{C}$ for 18 h. Measurement of antibiotic inhibition zones and interpretation of the data was carried out according to the British Society for Antimicrobial Chemotherapy guidelines (BSAC). Minimum inhibitory antibiotic concentrations for growth of $K$. sedentarius MBB13 was carried out in ISO broth medium in 96 well plates. Bacterial suspensions $\left(100 \mu \mathrm{L}\right.$ at $0.5 \mathrm{McF}$ arland standard; $\left.0.111 \mathrm{OD}_{600}\right)$ were added to ISO broth medium $(100 \mu \mathrm{L})$ containing $2,048 \mu \mathrm{g} \mathrm{mL}^{-1}$ of the indicated antibiotic. Then $100 \mu \mathrm{L}$ of this suspension was used in serial dilutions until a final concentration of $1 \mu \mathrm{g} \mathrm{mL} L^{-1}$. Data interpretation was according to the BSAC guidelines.

\section{Sample Preparation for Nuclear Magnetic Resonance (NMR)}

Overnight cultures (50 mL in $250 \mathrm{~mL}$ conical flasks) of $K$. sedentarius MBB13 were grown in Nutrient broth containing the indicated concentrations of $\mathrm{NaCl}$ at $37^{\circ} \mathrm{C}$ for $16 \mathrm{~h}$ with shaking $(250$ $\mathrm{rpm}$ ). Bacteria were collected by centrifugation for $10 \mathrm{~min}$ at 3,000 $g$. The supernatants were discarded and the pellets were resuspended in $1 \mathrm{~mL} \mathrm{dH_{2 }} \mathrm{O}$. The chilled bacterial suspensions were sonicated $(3 \times 20 \mathrm{~s}$ with $15 \mathrm{~s}$ cooling between each sonication step) to lyse the bacteria. Samples then were transferred to Eppendorf tubes and centrifuged at $13,000 \mathrm{~g}$ for $7 \mathrm{~min}$. The supernatants were transferred to pre-weighed Eppendorf tubes and frozen overnight at $-80^{\circ} \mathrm{C}$. The tubes were capped with lids that had been pierced with a needle and placed in a freeze-dryer for $72 \mathrm{~h}$. After re-capping with the original unpierced lids, the tubes were re-weighed and stored at room temperature. For analysis, the samples were dissolved in $530 \mu \mathrm{L} \mathrm{D} \mathrm{D}_{2} \mathrm{O}$ and $5 \mu \mathrm{L}$ trimethylsilyl propionate (100 $\mathrm{mM}$ ). NMR spectra were collected using a Bruker $600 \mathrm{MHz}$ instrument.

\section{Two-Hybrid Assay}

The bacterial adenylate cyclase-based two-hybrid (BACTH) system was used to detect interaction between WhiB proteins ( 1 , 2,3 , and 7 ) and sigma factors $(\mathrm{A}, \mathrm{B}, \mathrm{H}$, and $\mathrm{J})$ in vivo [Karimova et al., 1998]. The genes encoding the WhiB proteins and the sigma factors were amplified from via PCR using primers (online supplementary Table 1). PCR products were digested and ligated into pKT25 between the XbaI and KpnI for $w b l$ genes, and pUT18 between HindIII and KpnI for the sigma factors. Thus, each Wbl protein was fused to the C-terminal of T25, and each sigma factor was fused to the $\mathrm{N}$-terminal of T18 (online supplementary Table 3 and online supplementary Fig. 1). Due to amplification difficulties, whiB3, $\sigma^{\mathrm{A}} \mathrm{C}$-terminal domain (CTD), and $\sigma^{\mathrm{B}}$ were optimized to $E$. coli $\mathrm{K}-12$ and synthesised, and $\sigma^{\mathrm{J}}$ was synthesised and ligated into pUT18 by Eurofins (gene optimization was done using this webpage: http://gcua.schoedl.de/sequential_v2.html). Plasmids were propagated in E. coli DH5a. The plasmids that encode the T25WhiB and Sigma-T18 protein fusions were used to co-transform electrocompetent E. coli BTH101. Transformed cells were plated on LB agar supplemented with ampicillin $(100 \mu \mathrm{g} / \mathrm{mL})$, streptomy$\operatorname{cin}(50 \mu \mathrm{g} / \mathrm{mL})$, and kanamycin $(35 \mu \mathrm{g} / \mathrm{mL})$, and incubated at $37^{\circ} \mathrm{C}$ for 24-36 h. After electroporation, one colony was picked and transferred to LB broth $(5 \mathrm{~mL})$ containing appropriate antibiotics and incubated overnight at $37^{\circ} \mathrm{C}$. Samples $(10 \mu \mathrm{L})$ of the cultures were dropped onto MacConkey/maltose plates supplemented with maltose (1\%), the appropriate antibiotics, and IPTG $0.5 \mathrm{~mm}$ (for induction) and incubated at $30^{\circ} \mathrm{C}$ for $36-40 \mathrm{~h}$.

Purification of WhiB1 and WhiB2 Expressed in M. smegmatis

The coding regions of whiB1 and whiB2 were amplified from K. sedentarius MBB13 genomic DNA by PCR (online supplementary Table 1). The products were digested and ligated into pMyNT (Hygromycin-B "Hygro-BR") between the Ncol and HindIII sites for heterologous overproduction of the corresponding proteins in M. smegmatis $\mathrm{mc}^{2} 155$ (online supplementary Table 3 ). The integrity of both plasmids was checked by DNA sequencing. Plasmids containing whiB1 (JRG6949) and whiB2 (JRG6947) were purified and used to transform M. smegmatis mc $^{2} 155$ (JRG6161). Protein overproduction was carried out in $2 \mathrm{~L}$ flasks containing $7 \mathrm{H} 9$ medium with Hygro-BR $(100 \mu \mathrm{g} / \mathrm{mL})$. Cultures were grown at $37^{\circ} \mathrm{C}$ to $\mathrm{OD}_{600} \sim 0.9(\sim 18 \mathrm{~h})$ and then induced with acetamide $(0.2 \%)$ and incubated at $37^{\circ} \mathrm{C}$ for $\sim 12 \mathrm{~h}$, with shaking $(250 \mathrm{rpm})$. Cells were collected by centrifugation at $17,500 \mathrm{~g}$ for $30 \mathrm{~min}$ and stored at $-20^{\circ} \mathrm{C}$. Frozen cells were suspended in either $20 \mathrm{mM} \mathrm{NaH}_{2} \mathrm{PO}_{4}$, $\mathrm{pH} 7.4$, or $50 \mathrm{~mm}$ Tris- $\mathrm{HCl}, \mathrm{pH} 8.0$, containing $500 \mathrm{mM} \mathrm{NaCl}$, and disrupted by sonication. Cells were sonicated $(9 \times 25$ s pulses $)$ on ice by Soniprep 150 Plus set to the maximum amplitude. The sonicated samples were clarified by centrifugation at $45,500 \mathrm{~g}$ for 20 min. The His-tagged WhiB1 and WhiB2 proteins were isolated from the extracts $(10 \mathrm{~mL})$ by application to $5 \mathrm{~mL}$ HiTrap chelating 
Fig. 1. Genome comparison overview of K. sedentarius MBB13 and DSM 20547. a Linear diagram generated by the Galaxy assembly website (https://hactar.shef.ac. uk/galaxy). Arrows at the bottom indicate the position of contigs, red lines indicate conserved regions with the same polarity in both genomes, and blue lines indicate conserved regions with opposite polarity. b Circular diagram. The burgundy circle indicates the reference genome, and the green circle indicates the MBB13 genome. The \%GC content is shown in an adjacent feature track (black circle). The GC skew is shown in the last circle from inside (green and violet). This CGView map is generated via the online website of http://stothard. afns.ualberta.ca/cgview_server/ [Grant et al., 2012].

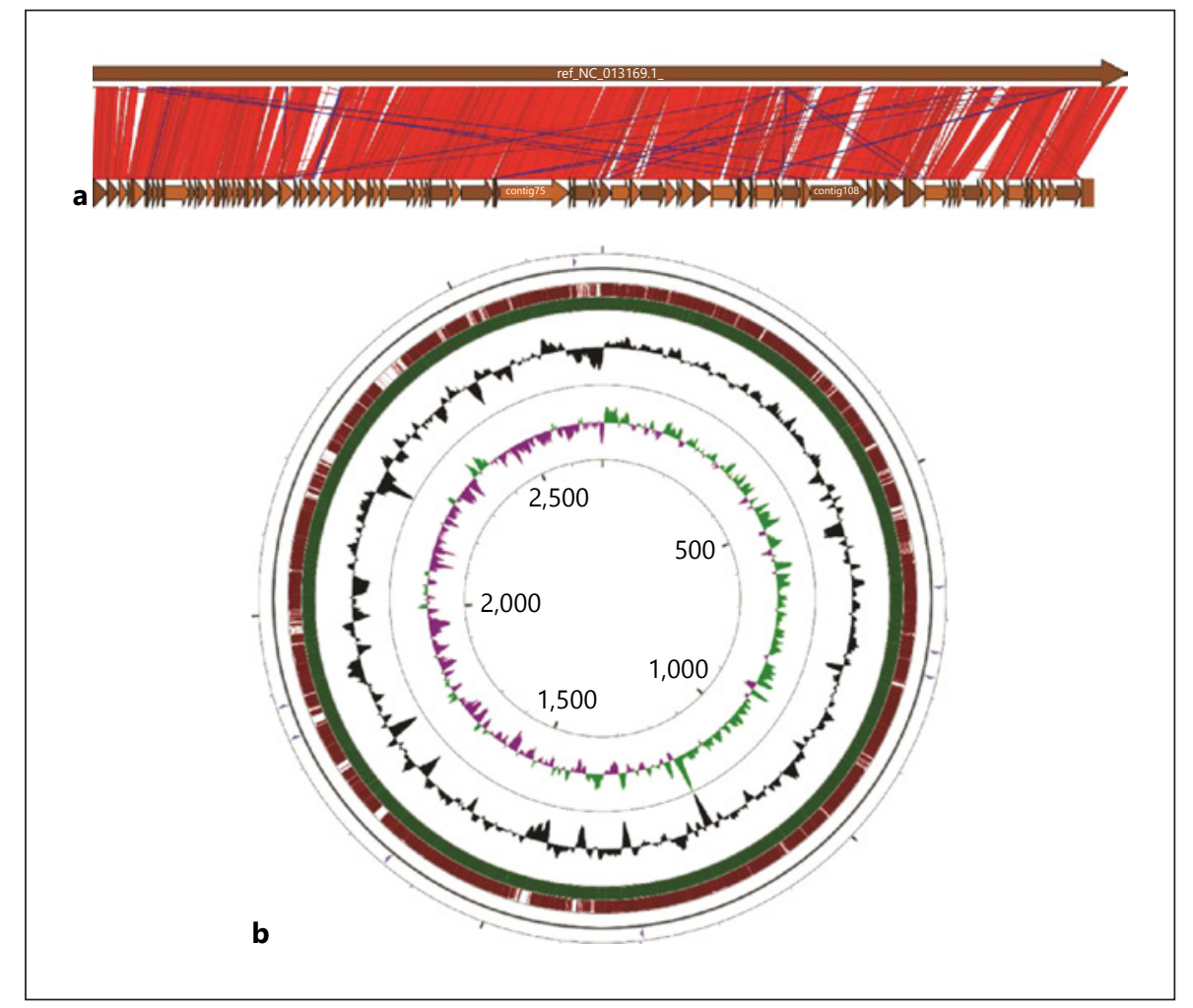

columns using the manufacturer's standard procedure with a linear gradient $(0-100 \%$ B) formed from binding buffer A $(50 \mathrm{~mm}$ Tris- $\mathrm{HCl}$, pH 8.0 containing $0.5 \mathrm{M} \mathrm{NaCl}$ ) and elution buffer $\mathrm{B}$ (buffer A containing $0.5 \mathrm{M}$ imidazole). Eluted fractions were analysed by SDS-polyacrylamide gel electrophoresis to determine purity and those containing $\mathrm{Wbl}$ proteins were stored at $4^{\circ} \mathrm{C}$.

\section{Protein Concentration Measurement}

Routinely, protein concentrations were measured by the Bradford assay (Bio-Rad) used according to the manufacturer's procedure. For pure proteins, the theoretical extinction coefficient of WhiB1 or WhiB2 was first obtained from the ExPASy webpage (https://web.expasy.org/protparam/), then absorbance at $280 \mathrm{~nm}$ was used to calculate the protein concentration.

\section{Iron-Sulfur Cluster Reconstitution}

All reconstitution steps were carried out inside the anaerobic cabinet (Don Whitley A85) at room temperature $\left(\sim 20^{\circ} \mathrm{C}\right)$ and $0 \%$ oxygen concentration. Buffers and other solutions were left for at least $18 \mathrm{~h}$ inside the cabinet to ensure that they were anaerobic. Iron-sulfur cluster reconstitution was achieved using the NifSbased system [Kudhair et al., 2017]. The reaction was left inside the anaerobic cabinet overnight. On the next day, the reconstituted WhiB2 was dialysed against anaerobic buffer: $25 \mathrm{~mm} \mathrm{NaH}{ }_{2} \mathrm{PO}_{4}$, $0.25 \mathrm{M} \mathrm{NaCl}, \mathrm{pH} 7.4$, overnight to remove non-integrated components. The dialysed, reconstituted WhiB2 was transferred into Hellma ${ }^{\circledR}$ quartz cuvette (10 mm pathlength) and the screw cap lid was sealed to maintain anaerobic conditions. The [4Fe-4S] cluster content was estimated by UV-visible spectroscopy using an extinction coefficient of $16,750 \mathrm{M}^{-1} \mathrm{~cm}^{-1}$ at $420 \mathrm{~nm}$ [Kudhair et al., 2017].
Reaction of WhiB1 and WhiB2 with NO

Spermine NONOate (Cayman) was used to investigate the sensitivity of the WhiB1 and WhiB2 iron-sulfur clusters to NO. Spermine NONOate was prepared by dissolving the powder in water to the final concentration of $19.06 \mathrm{~mm}$. Spermine NONOate solution $(30 \mu \mathrm{L})$ was injected using a Hamilton syringe into $1 \mathrm{~mL}$ of anaerobic protein $(\sim 35 \mu \mathrm{M})$ in a sealed Hellma ${ }^{\circledR}$ quartz cuvette. The reaction was followed by obtaining UV-visible spectra (300$800 \mathrm{~nm}$ ).

\section{Results}

The Genome Sequence of $K$. sedentarius $M B B 13$

The genome of $K$. sedentarius MBB13 was sequenced and assembled using the $K$. sedentarius DSM 20547 (541T) genome as a reference. The DNA was sequenced with 30x coverage and 152 contigs were used to assemble a 2,695,411 bp genome, which had a GC content of $71.57 \%$ (Fig. 1). The assembled genome was annotated using PROKKA [Seemann, 2014] and was predicted to contain 2,499 genes of which 1,789 were assigned a predicted function with the remaining 710 genes encoding hypothetical proteins (Table 1).

Although the $K$. sedentarius MBB13 genome should be considered to be a draft assembly, 85 loci present in the 
reference genome (K. sedentarius DSM 20547 (541T)) were unmatched in the MBB13 genome (online supplementary Table 6). Of these, 24 loci included genes coding for transposase/integrase family proteins, suggesting some element of horizontal gene acquisition. The unmatched regions contained 35 pseudogenes and 438 genes, of which 174 encoded either hypothetical or uncharacterised proteins. There were 27 unmatched transcriptional regulators and 4 unmatched $\sigma^{70}$-family sigma factors, suggesting that the unmatched loci incorporated at least some of the apparatus needed to integrate these genes into the core regulatory networks of $K$. sedentarius DSM 20547 (541T). K. sedentarius DSM 20547 (541T) has two $r r n$ operons coding for the 5, 16, and 23S rRNA molecules and one of these was apparently absent from the $K$. sedentarius MBB13 genome assembly. A notable feature of the unmatched genes was the presence of genes encoding multi-drug-resistance pumps ( 10 genes in 9 unmatched loci) and systems associated with resistance to $\mathrm{Cd}(\mathrm{II}), \mathrm{Hg}(\mathrm{II})$, and $\mathrm{Cu}(\mathrm{II})$.

The draft K. sedentarius MBB13 genome contained 79 loci made up of a total of 359 genes that were not matched in the reference genome (online supplementary Table 7). Of these, 181 genes coded for hypothetical proteins. Twelve of 79 unmatched loci contained predicted transposase/integrase family proteins (7 of each), suggesting that these regions were acquired horizontally. The unmatched regions included 18 genes coding for transcription regulators (with a further 3 helix-turn-helix domain proteins) and 2 sigma factors, including the extracytoplasmic function (ECF) sigma factor $\sigma^{J}$. There were no unambiguous candidates (such as erm genes coding for 23S rRNA methylation proteins) [Weisblum, 1995] within the unmatched regions to account for the observed antibiotic resistance profile of $K$. sedentarius MBB13. However, amongst the predicted transcription regulators was a two-component system, LiaS/VraR, which in other gram-positive bacteria coordinates the response to cell wall-targeting antibiotics [Kuroda et al., 2003; Shankar et al., 2015]. This two-component system sits upstream of genes coding for a predicted daunorubicin/doxorubicin resistance ATP-binding protein DrrA, two ABC-2 family transporter proteins, a hypothetical protein, and a VanZlike protein (online supplementary Table 7). Drr-type ABC-transporters contribute to moderate level macrolide resistance in $M$. tuberculosis complex bacteria [Choudhuri et al., 2002], and so this locus could be involved in the observed erythromycin resistance. Furthermore, VanZ membrane proteins are associated with resistance to lipoglycopeptide antibiotics [Vimberg et al.,
Table 1. Overall genome sequence composition of $K$. sedentarius MBB13 and DSM 20547

\begin{tabular}{lrr}
\hline & MBB13 & DSM 20547 \\
\hline Number of bases & $2,695,411$ & $2,785,024$ \\
GC percentage & 71.55 & 71.63 \\
Total genes & 2,499 & 2,554 \\
Assigned genes & 1,789 & 1,963 \\
Genes encoding hypothetical proteins & 710 & 591 \\
\hline
\end{tabular}

2020]. Interestingly, there is a second Drr-type system in K. sedentarius MBB13 that is apparently absent in DSM 20547 (541T). Other potential contributors to the observed antibiotic resistance profile of $K$. sedentarius MBB13 included a predicted streptothricin hydrolase and the multidrug efflux pumps Stp and QacA. The former could expand the range of antibiotic resistance by inactivating streptothricin and the latter by efflux of antibiotics. To determine the contributions, if any, of these unmatched genes to the observed resistance of $K$. sedentarius MBB13 to ciprofloxacin, erythromycin, and/or gentamicin will require further experiments with mutant strains.

\section{K. sedentarius MBB13 Is an Aerobe}

The $K$. sedentarius type strain is an obligate aerobe [Sims et al., 2009]. Whereas the facultative anaerobe $E$. coli exhibited growth throughout a thioglycollate broth medium, $K$. sedentarius MBB13 grew only at the surface in contact with air, indicating that $K$. sedentarius MBB13 is an aerobe (Fig. 2a). This conclusion was supported by assessing $K$. sedentarius MBB13 growth on solid medium under anaerobic and aerobic conditions (Fig. 2b). Analysis of the genome sequence indicated the presence of a complete glycolytic pathway and citric acid cycle, but the absence of genes associated with anaerobic fermentative or respiratory metabolism. Some strains of $K$. sedentarius are able to reduce nitrate to nitrite [Sims et al., 2009], a reaction which could allow nitrate to serve as an alternative terminal electron acceptor; however, the MBB13 strain did not possess genes resembling known nitrate reductases. In contrast, K. sedentarius MBB13 appeared to have genes coding for a branched aerobic respiratory chain beginning with $\mathrm{NADH}$, succinate, and proline dehydrogenases and ending with cytochrome $b c 1-a a 3$ and cytochrome $b d$ oxidases, linked by the quinone MK8-12, to permit $\mathrm{O}_{2}$ to be used as the terminal electron acceptor (Fig. 2c). 
Fig. 2. K. sedentarius MBB13 is an aerobe. a Growth of $K$. sedentarius MBB13 was only observed at the interface between thioglycollate broth and the atmosphere (left), whereas growth of the facultative anaerobe, E. coli MG1655, was observed within the broth (right). b Growth of K. sedentarius MBB13 on LB agar under anaerobic (left) and aerobic (right) conditions. c $K$. sedentarius $\mathrm{MBB} 13$ has genes coding for a branched aerobic electron transport chain. The primary dehydrogenases, NADH $(\mathrm{Ndh})$, succinate $(\mathrm{Sdh})$, and proline (PutP/ ProC) and their predicted $\mathrm{H}^{+} / \mathrm{e}^{-}$ratios are shown, along with the quinone (MK8-12) and the terminal oxidases cytochrome $b c 1$ $a a 3$ and cytochrome $b d$ and their predicted $\mathrm{H}^{+} / \mathrm{e}^{-}$ratios.
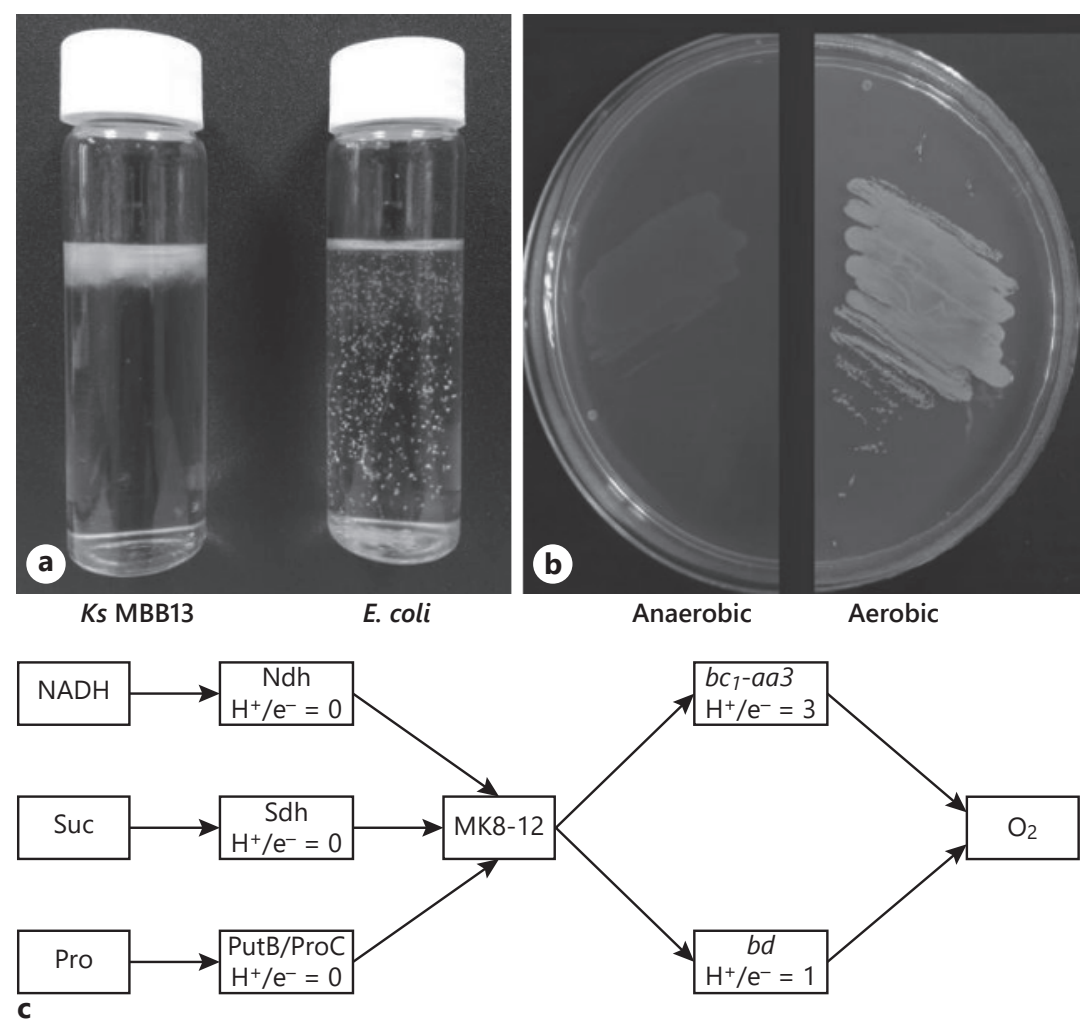

Glycine Betaine Is the Major Compatible Solute of

K. sedentarius $M B B 13$

The $K$. sedentarius type strain $541 \mathrm{~T}$ can grow in media containing up to $10 \%(\sim 1.7 \mathrm{M}) \mathrm{NaCl}$ [Sims et al., 2009]. The $\mathrm{OD}_{600}$ measurements of $K$. sedentarius MBB13 cultured for $24 \mathrm{~h}$ in Nutrient broth supplemented with additional $\mathrm{NaCl}$ (nutrient broth contains $0.1 \mathrm{M} \mathrm{NaCl}$ ) showed that growth negatively correlated with increased salinity (Fig. 3a). At $\mathrm{NaCl}$ concentrations $>1.1 \mathrm{M}$ growth was weak, suggesting that $K$. sedentarius MBB13 is only moderately halotolerant [Ara et al., 2013]. Many bacteria use compatible solutes to counteract the effects of high osmolarity environments [Wood et al., 2001]. Proton NMR analysis of cell extracts from $K$. sedentarius MBB13 cultures grown for $24 \mathrm{~h}$ in the presence of different $\mathrm{NaCl}$ concentrations showed that glycine betaine accumulated as the $\mathrm{NaCl}$ concentration increased (Fig. 3b). Proline appeared in the NMR spectra at $\mathrm{NaCl}$ concentrations $>1.1$ M. A species with chemical shift of $\sim 4.1 \mathrm{ppm}$ suggested that ectoine was produced when the $\mathrm{NaCl}$ concentration was $>2.1 \mathrm{M}$.

Choline is a precursor of glycine betaine. Choline and glycine betaine uptake is mediated by the ABC-type transporter OpuC, which is likely encoded by the opuBAopuCD-opuCB-opuCC-lcdH operon in the K. sedentarius $\mathrm{MBB} 13$ genome; the opuD and bet $L$ genes, coding for Betaine/Carnitine/Choline Transporter (BCCT) family transporters, could also permit uptake of these solutes. In gram-positive bacteria conversion of choline to glycine betaine is a two-step process catalysed by choline dehydrogenase, GbsB and an aldehyde dehydrogenase, BetB [Wood et al., 2001; Wood, 2006]. Although a betB gene was identified in the $K$. sedentarius MBB13 genome, a $g b s B$ ortholog was not detected. Ectoine is a major compatible solute in many halophiles, $K$. sedentarius MBB13 possesses an ask-ect-asd-ect $A B C D$ operon and thus has the necessary machinery for synthesis of the precursor, L-aspartate- $\beta$-semialdehyde (ask-ect-asd), and its subsequent conversion to ectoine/hydroxyectoine (ect $A B C D$ ) (Fig. 3c). Proline and trehalose are widely used compatible solutes and $K$. sedentarius MBB13 possesses a proline transporter (proP) and trehalose synthesis enzymes (ots $A B)$ [Kaasen et al., 1994] (Fig. 3c). Following osmotic down-shifts these accumulated compatible solutes need to be released to minimise water influx and $K$. sedentarius $\mathrm{MBB} 13$ has two mechanosensitive channels 


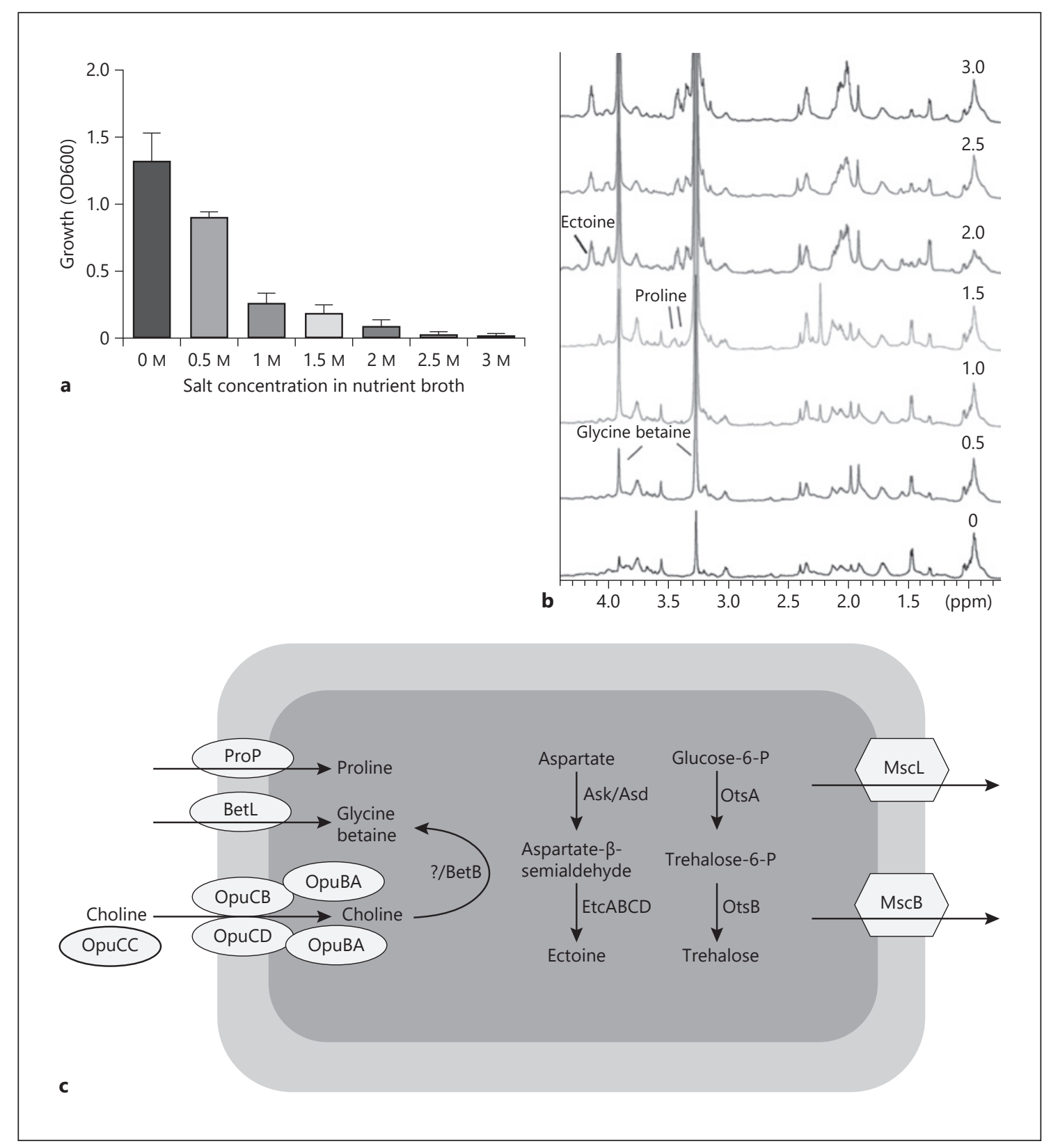

Fig. 3. K. sedentarius MBB13 systems involved in osmotic adaptation. a Growth of $K$. sedentarius MBB13 in nutrient broth supplemented with different concentrations of $\mathrm{NaCl}$ at $\mathrm{pH} 7.5 \pm 0.2$ after $24 \mathrm{~h}$ incubation at $37^{\circ} \mathrm{C}$ on an orbital shaker (150 rpm). Optical density was measured at $600 \mathrm{~nm}\left(\mathrm{OD}_{600}\right)$ against a medium blank. Data points are the means of three replicates plus or minus standard deviation. b Proton NMR spectra of cell extracts from $K$. sedentarius MBB13 grown in nutrient broth medium (containing 0.1 $\mathrm{M} \mathrm{NaCl}$ ) supplemented with $0,0.5,1.0,1.5,2.0,2.5$, and $3.0 \mathrm{M} \mathrm{NaCl}$. The spectra shown are equivalent to $\sim 10 \mathrm{OD}_{600}$ units of bacteria without additional $\mathrm{NaCl}, \sim 8 \mathrm{OD}_{600}$ units of bacteria for cultures supplemented with $0.5,1.0$, or $1.5 \mathrm{M} \mathrm{NaCl}, \sim 6 \mathrm{OD}_{600}$ units of bac- teria for cultures supplemented with $2.0 \mathrm{M} \mathrm{NaCl}$, and $0.2 \mathrm{OD}_{600}$ units of bacteria for cultures supplemented with $3.0 \mathrm{M} \mathrm{NaCl}$. Peaks associated with relevant compounds are indicated. c Analysis of the genome sequence identified transporters, channels, and biosynthetic pathways that could contribute to tolerating high salinity. Membrane proteins involved in release or uptake of environmental compatible solutes and cytoplasmic enzymes for the synthesis of compatible solutes (glycine betaine, ectoine, and trehalose) are shown. MscS and MscL are mechanosensitive channels which are transient gating systems to release compatible solutes in response to an osmotic down-shock, preventing cell lysis. 


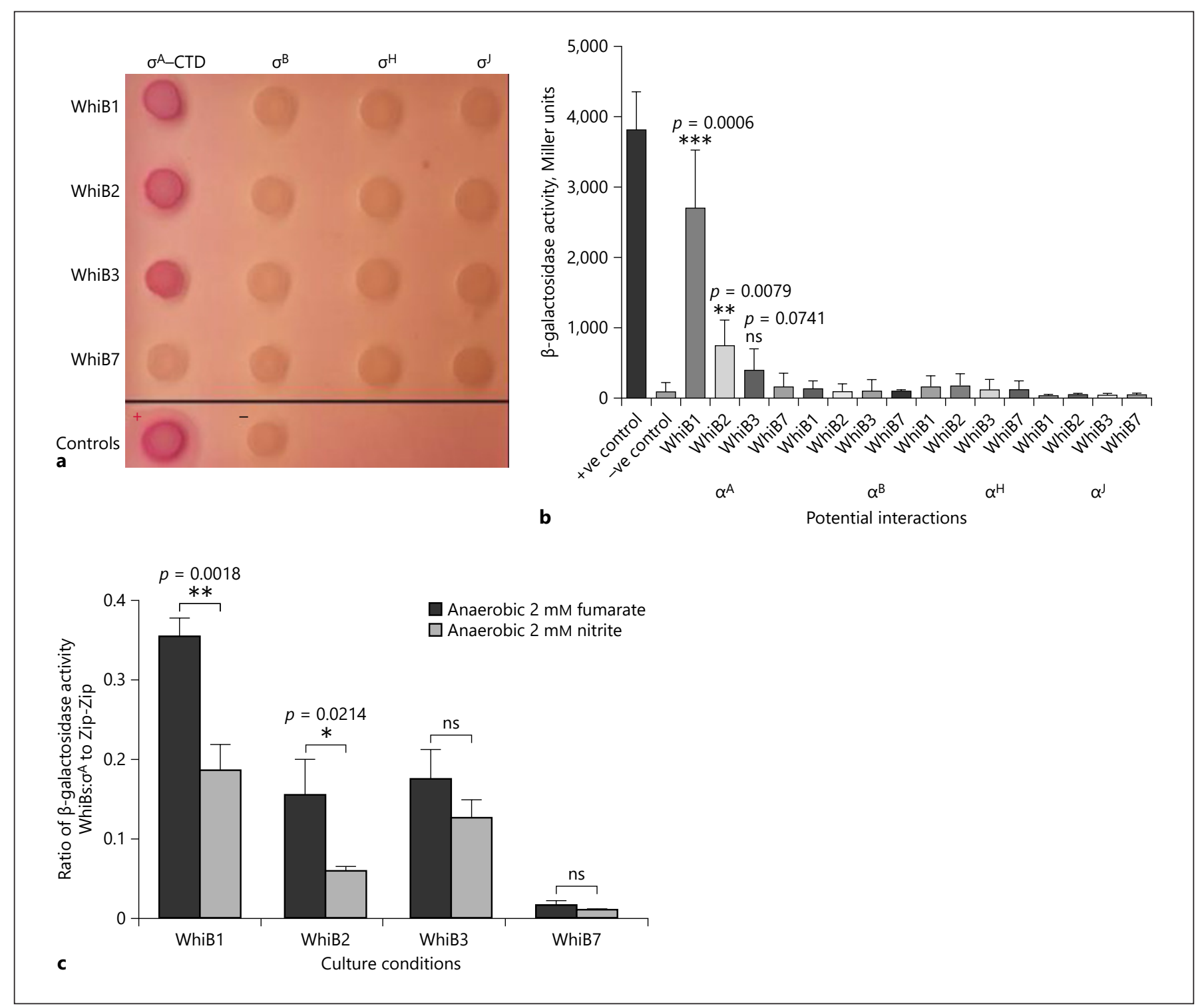

Fig. 4. Interaction of $\mathrm{Wbl}$ proteins and sigma factors $(\sigma)$ using the BATCH system. a Patches of E. coli BTH101 co-transformed with pKT-25 and pUT18 coding for the indicated fusion proteins on MacConkey medium in the presence of maltose. The pKT25-zip and pUT18-zip plasmids served as a positive control (+) and the pKT25 and pUT18 vectors served as a negative control (-) (bottom row). b Interaction between fusion partners in liquid cultures. Data points are the mean values for $\beta$-galactosidase specific ac-

( $m s c L$ and $y k u T / m s c S)$ to fulfil this function. Thus, analysis of the $K$. sedentarius MBB13 genome and the experiments presented here suggest that glycine betaine is the major compatible solute, but proline and ectoine also contribute to tolerance at higher $\mathrm{NaCl}$ concentrations. tivities from four cultures, plus or minus standard deviation; significance $\left(t\right.$ test) is indicated. c Nitric oxide impairs the WhiB1: $\sigma^{A}$ and WhiB2: $\sigma^{A}$ interactions. The data shown are $\beta$-galactosidase specific activities normalised to those obtained for the positive control (Zip-Zip) cultures grown under the indicated conditions. The chart shows the means and standard deviations for four independent cultures; significance ( $t$ test) is indicated.

\section{K. sedentarius MBB13 Has Four Wbl Proteins That}

Interact with the Primary Sigma Factor, $\sigma^{A}$

One of the characteristics of actinobacteria is the possession of Wbl (WhiB-like) proteins [Bush, 2018]. Wbl proteins are small ( $\sim 80-140$ residues) with four invariant cysteine residues that coordinate a $[4 \mathrm{Fe}-4 \mathrm{~S}]$ cluster [Bush, 

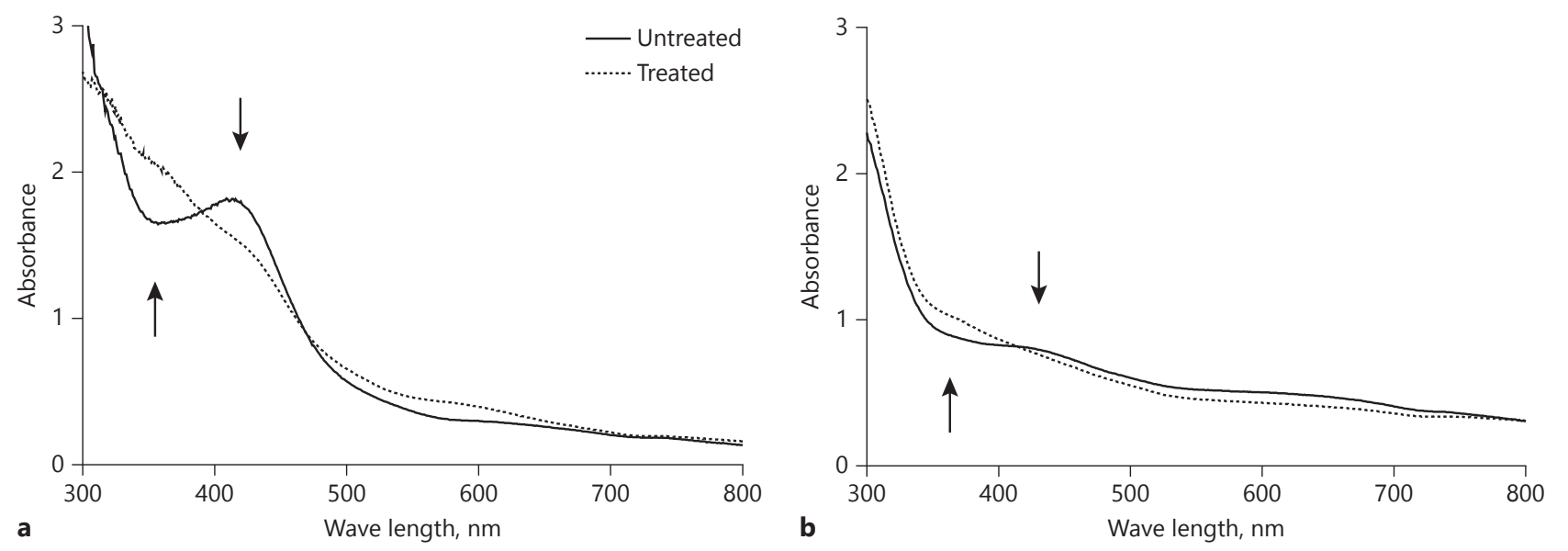

Fig. 5. The iron-sulfur clusters of K. sedentarius MBB13 WhiB1 and WhiB2 proteins react with nitric oxide. a UV-visible spectra of holo-WhiB1 ( $58 \mu \mathrm{M}$ cluster) before (solid line) and after (dashed line) exposure to Spermine NONOate $(571.8 \mu \mathrm{M})$. b UV-visible spectra of WhiB2 $(25 \mu \mathrm{M}$ cluster) before (solid line) and after (dashed line) treatment with Spermine NONOate $(571.8 \mu \mathrm{M})$. The arrows indicate the direction of the absorbance changes. The treated traces were normalised to the untreated readings.

2018]. The iron-sulfur cluster acts as a redox sensor in controlling diverse biological processes, such as morphogenesis, metabolism, virulence, and antibiotic resistance, most likely by interacting with, and modulating the activity of, RNA polymerase sigma factors [Miller and Auerbuch, 2015; Kudhair et al., 2017; Wan et al., 2020]. K. sedentarius $\mathrm{MBB} 13$ possesses four $\mathrm{Wbl}$ encoding genes (whiB1, whiB2, whiB3, and whiB7) and four RNA polymerase sigma factors ( $\operatorname{sig} A, \operatorname{sig} B, \operatorname{sigH}$, and sigJ) (online supplementary Fig. 2). All possible interactions between the $\mathrm{Wbl}$ proteins and sigma factors were tested using a bacterial two-hybrid (BACTH) system [Karimova et al., 1998]. Plating on indicator medium suggested that the WhiB1, B2, and B3 proteins interacted with the C-terminal domain of $\sigma^{\mathrm{A}}$, but not with any of the other sigma factors; WhiB7 did not interact with any of the sigma factors (Fig. 4a). This conclusion was confirmed by measuring $\beta$-galactosidase activities of aerobic liquid cultures, although the WhiB3: $\sigma^{\mathrm{A}}$ interaction, whilst greater than the negative control $(p=0.0741)$, was weaker than in other protein combinations (Fig. 4b). Although these data suggest that the K. sedentarius MBB13 Wbl proteins only interact with $\sigma^{\mathrm{A}}$, the possibility that the $\sigma^{\mathrm{B}}-\mathrm{T} 18, \sigma^{\mathrm{H}}-\mathrm{T} 18$, and/or $\sigma^{\mathrm{J}}-\mathrm{T} 18$ fusion proteins were incorrectly folded and/or unstable, leading to false negative results, cannot be excluded.

Previous work had shown that the interaction between M. tuberculosis WhiB1 and $\sigma^{\mathrm{A}}$ was impaired in the pres- ence of nitric oxide [Kudhair et al., 2017]. This was also the case for the $K$. sedentarius MBB13 WhiB1: $\sigma^{\mathrm{A}}$ and WhiB2: $\sigma^{\mathrm{A}}$ interactions (Fig. 4c). Recombinant expression and purification of the K. sedentarius MBB13 WhiB1 and WhiB2 proteins showed that the WhiB1 iron-sulfur cluster was stable under aerobic conditions, as judged by the colour of the isolated protein (straw brown) and its UVvisible spectrum being typical of a [4Fe-4S] protein (Fig. 5a). In contrast, the isolated WhiB2 protein was colourless and after anaerobic reconstitution the UV-visible spectrum indicated the presence of both $[4 \mathrm{Fe}-4 \mathrm{~S}]$ and [2Fe-2S] clusters, suggesting that the WhiB2 iron-sulfur cluster was less stable than that of WhiB1 (Fig. 5b). Consistent with the impaired interaction observed using the BACTH system, both WhiB1 and WhiB2 iron-sulfur clusters reacted with nitric oxide with changes in the UVvisible spectra (increased absorbance at 350-360 nm and decreased absorbance at $420 \mathrm{~nm}$ ) suggesting the formation of a dinuclear iron dinitrosyl species known as Roussin's red ester [Kudhair et al., 2017; Crack and Le Brun, 2019] (Fig. 5).

\section{Discussion}

A strain of $K$. sedentarius (designated as MBB13) isolated from a dehumidifier operating in a university lecture theatre was found to be resistant to three antibiotics (cip- 
rofloxacin, erythromycin, and gentamicin; online supplementary Tables 4 and 5). Inspection of the $K$. sedentarius MBB13 draft genome suggested several possible contributors to this antibiotic resistance profile, including several efflux pumps, without revealing the source of the resistance to erythromycin, which was of particular interest because this antibiotic is often used to treat $K$. sedentarius infections. The isolated strain was an aerobe, with genes suggesting a central metabolic map consisting of glycolysis, citric acid cycle, and a branched aerobic electron transport chain. Some K. sedentarius strains have been reported to reduce nitrate, raising the possibility of anaerobic nitrate respiratory growth, but nitrate reductase genes were absent from the $K$. sedentarius MBB13 genome. $K$. sedentarius is found on the skin, mucosae, and oropharynx of human beings [Sims et al., 2009] and this is likely to be the source of the strain trapped by the dehumidifier. K. sedentarius MBB13 possessed several mechanisms for counteracting osmotic stress, including compatible solute uptake systems and enzymes for synthesis of ectoine and trehalose (Fig. 3c). It appeared that glycine betaine was the major compatible solute, but was supplemented at higher salt concentrations by ectoine and proline.

One of the characteristics of actinobacteria is the possession of Wbl proteins [Feng et al., 2016]. K. sedentarius MBB13 has four $w b l$ genes similar to the whiB1, whiB2, whiB3, and whiB7 of M. tuberculosis (online supplementary Fig. 2). The major M. tuberculosis sigma factor $\left(\sigma^{\mathrm{A}}\right)$ interacts with all the $M$. tuberculosis Wbl proteins except WhiB5 [Feng et al., 2016]. K. sedentarius MBB13 has four sigma factors $\left(\sigma^{\mathrm{A}}, \sigma^{\mathrm{B}}, \sigma^{\mathrm{H}}\right.$, and $\left.\sigma^{\mathrm{J}}\right)$ (online supplementary Fig. 2). Therefore, BACTH system was used to test all possible interactions between the K. sedentarius MBB $13 \mathrm{Wbl}$ proteins and the sigma factors. Significant interactions were identified for WhiB1 and WhiB2 with $\sigma^{\mathrm{A}}$ (Fig. 4). The interaction of $M$. tuberculosis of WhiB1 with $\sigma^{\mathrm{A}} \mathrm{de}-$ pends on the presence of the WhiB1 [4Fe-4S] cluster [Kudhair et al., 2017; Wan et al., 2020]. In M. tuberculosis, $\sigma^{\mathrm{A}}{ }_{\text {СтD }}$ inserts His516 and Pro517 of the C-terminal loop into the binding pocket of the WhiB $1[4 \mathrm{Fe}-4 \mathrm{~S}]$ cluster to initiate the interaction, while in WhiB1, the binding pocket is a cluster of hydrophobic residues including Trp3, Phe17, Trp60, and Phe18, which are highly conserved in WhiB1 cross actinobacteria [Wan et al., 2020]; those residues are also conserved in K. sedentarius MBB13 WhiB1 (online supplementary Fig. 3). Therefore, the mechanism of WhiB1: $\sigma^{\mathrm{A}}{ }_{\text {CTD }}$ interaction is likely to be conserved in K. sedentarius MBB13 and M. tuberculosis.

Nitric oxide reacted with the [4Fe-4S] clusters of the WhiB1 and WhiB2 proteins (Fig. 5) and the BACTH inter- actions were sensitive to conditions that generated NO, suggesting that $K$. sedentarius MBB13 WhiB1 and WhiB2 alter $\sigma^{\mathrm{A}}$ function in response to nitric oxide. Strains of $K$. sedentarius that possess a nitrate reductase could be exposed to endogenous nitric oxide, generated by nitrite reduction. However, K. sedentarius MBB13 lacks a nitrate reductase and hence WhiB1 is more likely to respond to exogenous nitric oxide, perhaps generated by other microbes or the host immune system. Furthermore, the ironsulfur cluster of $K$. sedentarius MBB13 WhiB2 appeared to be oxygen-sensitive and so could act as a sensor of oxygen availability, an important parameter for an aerobe, rather than nitric oxide. Further research will be needed to test this speculation and addresses the open questions, including: why are multiple $\mathrm{Wbl}$ proteins present, how do WhiB1 and WhiB2 (and possibly WhiB3) alter gene expression though their interactions with $\sigma^{\mathrm{A}}$, and what is the primary signal(s) that cause disassembly of the $\mathrm{Wbl}-\sigma^{\mathrm{A}}$ complexes?

\section{Acknowledgments}

We thank Professor Milton Wainwright (University of Sheffield) for initiating the project, and Dr Joseph Webb and Dr James Gilmour (University of Sheffield) for useful discussions and advice on experimental design and Dr Andrea Hounslow (University of Sheffield) for assistance with NMR experiments, and Professor David Kelly (University of Sheffield) for access to laboratory equipment.

\section{Statement of Ethics}

The authors declare that the experiments reported were compliant with the regulations overseen by the University of Sheffield Biosafety Committee under the following reference numbers: GMO1984_01 and GMO2010_03A.

\section{Conflict of Interest Statement}

The authors declare that they have no competing interests.

\section{Funding Sources}

This work was supported by the Royal Embassy of Saudi Arabia Cultural Bureau in London and Saudi Food and Drug Authority (SFDA) through a $\mathrm{PhD}$ scholarship to M.A.A.

\section{Author Contributions}

J.G. and M.A.A. conceived the study. M.A.A., J.G. and B.K.K. performed the experiments. M.A.A. and J.G. drafted the manuscript. All authors read and approved the final manuscript. 


\section{References}

Ara I, Daram D, Baljinova T, Yamamura $\mathrm{H}$, Hozzein W, Bakir M, et al. Isolation, classification, phylogenetic analysis and scanning electron microscopy of halophilic, halotolerant and alkaliphilic actinomycetes isolated from hypersaline soil. Afr J Microbiol. Res.. 2013 Jan;7(4):298-308.

Budi SW, van Tuinen D, Martinotti G, Gianinazzi S. Isolation from the Sorghum bicolor Mycorrhizosphere of a bacterium compatible with Arbuscular Mycorrhiza development and antagonistic towards soilborne fungal pathogens. Appl Environ Microbiol. 1999 Nov;65(11):5148-50.

Burian J, Yim G, Hsing M, Axerio-Cilies P, Cherkasov A, Spiegelman GB, et al. The mycobacterial antibiotic resistance determinant WhiB7 acts as a transcriptional activator by binding the primary sigma factor SigA (RpoV). Nucleic Acids Res. 2013 Dec;41(22):10062-76.

Bush MJ. The actinobacterial WhiB-like (Wbl) family of transcription factors. Mol Microbiol. 2018 Aug;110(5):663-76.

Carver TJ, Rutherford KM, Berriman M, Rajandream MA, Barrell BG, Parkhill J. ACT: the Artemis comparison tool. Bioinformatics. 2005 Jun;21(16):3422-3.

Casonato S, Cervantes Sánchez A, Haruki H, Rengifo González M, Provvedi R, Dainese E, et al. WhiB5, a transcriptional regulator that contributes to Mycobacterium tuberculosis virulence and reactivation. Infect Immun. 2012 Sep;80(9):3132-44.

Chawla M, Parikh P, Saxena A, Munshi M, Mehta M, Mai D, et al. Mycobacterium tuberculosis WhiB4 regulates oxidative stress response to modulate survival and dissemination in vivo. Mol Microbiol. 2012 Sep;85(6):1148-65.

Chen Z, Hu Y, Cumming BM, Lu P, Feng L, Deng J, et al. Mycobacterial WhiB6 differentially regulates ESX-1 and the Dos regulon to modulate granuloma formation and virulence in Zebrafish. Cell Rep. 2016 Aug 30;16(9):2512-24.

Choudhuri BS, Bhakta S, Barik R, Basu J, Kundu $\mathrm{M}$, Chakrabarti P. Overexpression and functional characterization of an ABC (ATPbinding cassette) transporter encoded by the genes drrA and drrB of Mycobacterium tuberculosis. Biochem J. 2002;367(Pt 1):279-85.

Crack JC, Le Brun NEL. Mass Spectrometric Identification of $[4 \mathrm{Fe}-4 \mathrm{~S}](\mathrm{NO}) \mathrm{x}$ Intermediates of Nitric Oxide Sensing by Regulatory Iron-Sulfur Cluster Proteins. Chemistry. 2019 Mar 7;25(14):3675-84.

Feng L, Chen Z, Wang Z, Hu Y, Chen S. Genomewide characterization of monomeric transcriptional regulators in Mycobacterium tuberculosis. Microbiology (Reading, Engl). 2016 Feb;162(5):889-97.

Fujiyoshi S, Tanaka D, Maruyama F. Transmission of airborne bacteria across built environments and its measurement standards: A review. Front Microbiol. 2017 Nov 29;8:2336.

Gomez JE, Bishai WR. whmD is an essential mycobacterial gene required for proper septation and cell division. Proc Natl Acad Sci USA. 2000 Jul 18;97(15):8554-9.

Grant JR, Arantes AS, Stothard P. Comparing thousands of circular genomes using the CGView Comparison Tool. BMC Genomics. 2012 May;13(1):202.

Greene GR, Ninch CM, Foltz EL. Cerebral cyst infection with Micrococcus sedentarius: Case report. J Neurosurg. 1980 Jan;52(1):126-8.

Kaasen I, McDougall J, Strøm AR. Analysis of the otsBA operon for osmoregulatory trehalose synthesis in Escherichia coli and homology of the OtsA and OtsB proteins to the yeast trehalose-6-phosphate synthase/phosphatase complex. Gene. 1994 Mar;145(1):9-15.

Karimova G, Pidoux J, Ullmann A, Ladant D. A bacterial two-hybrid system based on a reconstituted signal transduction pathway. Proc Natl Acad Sci USA. 1998 May;95(10):5752-6.

Kudhair BK, Hounslow AM, Rolfe MD, Crack JC, Hunt DM, Buxton RS, et al. Structure of a Wbl protein and implications for NO sensing by M. tuberculosis. Nat Commun. 2017 Dec; $8(1): 2280$

Kuroda M, Kuroda H, Oshima T, Takeuchi F, Mori H, Hiramatsu K. Two-component system VraSR positively modulates the regulation of cell-wall biosynthesis pathway in Staphylococcus aureus. Mol Microbiol. 2003 Aug;49(3):807-21.

Lee JH, Yoo JS, Kim Y, Kim JS, Lee EJ, Roe JH. The WblC/WhiB7 transcription factor controls intrinsic resistance to translation-targeting antibiotics by altering ribosome composition. mBio. 2020 Apr 14;11(2):e00625-20.

Levenga H, Donnelly P, Blijlevens N, Verweij P, Shirango H, de Pauw B. Fatal hemorrhagic pneumonia caused by infection due to Kytococcus sedentarius---a pathogen or passenger? Ann Hematol. 20042003 Dec;83(7):447-9.

Longshaw CM, Wright JD, Farrell AM, Holland KT. Kytococcus sedentarius, the organism associated with pitted keratolysis, produces two keratin-degrading enzymes. J Appl Microbiol. 2002 Jul;93(5):810-6.

Miller HK, Auerbuch V. Bacterial iron-sulfur cluster sensors in mammalian pathogens. Metallomics. 2015 Feb;7(6):943-56.

Mnif B, Boujelbène I, Mahjoubi F, Gdoura R, Trabelsi I, Moalla S, et al. Endocarditis due to Kytococcus schroeteri: case report and review of the literature. J Clin Microbiol. 2006 Mar; 44(3):1187-9.

Napoli C, Marcotrigiano V, Montagna MT. Air sampling procedures to evaluate microbial contamination: a comparison between active and passive methods in operating theatres. BMC Public Health. 2012 Aug 2;12:594.

Osman S, La Duc MT, Dekas A, Newcombe D, Venkateswaran K. Microbial burden and diversity of commercial airline cabin air during short and long durations of travel. ISME J. 2008 Feb;2(5):482-97.

Pranteda G, Carlesimo M, Pranteda G, Abruzzese C, Grimaldi M, De Micco S, et al. Pitted kera- tolysis, erythromycin, and hyperhidrosis. Dermatol Ther. 2014;27(2):101-4.

Sader HS, Jones RN. Antimicrobial susceptibility of uncommonly isolated non-enteric Gramnegative bacilli. Int J Antimicrob Agents. 2005 Feb;25(2):95-109.

Savini V, Catavitello C, Masciarelli G, Astolfi D, Balbinot A, Bianco A, et al. Drug sensitivity and clinical impact of members of the genus Kocuria. J Med Microbiol. 2010 Dec;59(Pt 12):1395-402.

Seemann T. Prokka: rapid prokaryotic genome annotation. Bioinformatics. 2014 Jul;30(14): 2068-9..Prokka

Shankar M, Mohapatra SS, Biswas S, Biswas I. Gene regulation by the LiaSR two-component system in Streptococcus mutans. PLoS One. 2015;10(5): e0128083.

Sims D, Brettin T, Detter JC, Han C, Lapidus A, Copeland A, et al. Complete genome sequence of Kytococcus sedentarius type strain (541T). Stand Genomic Sci. 2009 Jul;1(1):12.

Singh A, Guidry L, Narasimhulu KV, Mai D, Trombley J, Redding KE, et al. Mycobacterium tuberculosis WhiB3 responds to $\mathrm{O} 2$ and nitric oxide via its [4Fe-4S] cluster and is essential for nutrient starvation survival. Proc Natl Acad Sci USA. 2007 Jul 10;104(28):11562-7.

Smith LJ, Stapleton MR, Fullstone GJ, Crack JC, Thomson AJ, Le Brun NE, et al. Mycobacterium tuberculosis WhiB1 is an essential DNA-binding protein with a nitric oxide-sensitive iron-sulfur cluster. Biochem J. 2010 Dec 15;432(3):417-27.

Stackebrandt E, Koch C, Gvozdiak O, Schumann P. Taxonomic dissection of the genus Micrococcus: Kocuria gen. nov., Nesterenkonia gen. nov., Kytococcus gen. nov., Dermacoccus gen. nov., and Micrococcus Cohn 1872 gen. emend. Int J Syst Bacteriol. 1995 Oct; 45(4):682-92.

Tille PM. Bailey and Scott's Diagnostic Microbiology. Maryland Heights: Mosby Inc.; 2014.

Vimberg V, Zieglerová L, Buriánková K, Branny P, Balíková Novotná G. VanZ reduces the binding of lipoglycopeptide antibiotics to Staphylococcus aureus and Streptococcus pneumoniae cells. Front Microbiol. 2020 Apr 3;11:566.

Wan T, Li S, Beltran DG, Schacht A, Zhang L, Becker DF, et al. Structural basis of non-canonical transcriptional regulation by the $\sigma \mathrm{A}$-bound iron-sulfur protein WhiB1 in M. tuberculosis. Nucl Acids Res. 2020 Jan;48(2):501-16.

Weisblum B. Erythromycin resistance by ribosome modification. Antimicrob Agents Chemother. 1995 Mar;39(3):577-85.

Wood JM. Osmosensing by bacteria. Sci STKE. 2006 Oct2006;2006(357):pe43-pe43.

Wood JM, Bremer E, Csonka LN, Kraemer R, Poolman B, van der Heide T, et al. Osmosensing and osmoregulatory compatible solute accumulation by bacteria. Comp Biochem Physiol, Part A Mol Integr Physiol. 2001 Oct; 130(3):437-60. 